\title{
Effect of a finite-range impurity potential on two-dimensional Anderson localization
}

\author{
M. T. Béal-Monod, A. Theumann, ${ }^{*}$ and G. Forgacs ${ }^{\dagger}$ \\ Laboratoire de Physique des Solides, Bâtiment 510, Université Paris-Sud, 91405 Orsay CEDEX, France
}

(Received 19 May 1992)

\begin{abstract}
We show that in two-dimensional weakly disordered metals a finite-range impurity-scattering potential reinforces the localization of the electronic states.
\end{abstract}

\section{INTRODUCTION}

We reexamine the conductivity of weakly disordered two-dimensional (2D) metals containing a small amount of randomly spread nonmagnetic impurities. Within the hypothesis of noninteracting electrons and independent impurities, the key result is well known. When the impurity concentration increases so that the Anderson transition is approached from the metallic side, the electron system is localized in dimensions of $d \leq 2$, whatever the degree of disorder.

In the present paper, we consider a system of noninteracting electrons and independent impurities and we also use a perturbation expansion in $\left(\epsilon_{F} \tau\right)^{-1}$ in the weakly localized regime $\left(\epsilon_{F} \tau\right) \gg 1, \epsilon_{F}$ and $\tau$ being the Fermi energy and the electron elastic lifetime, respectively. Instead of the usual ${ }^{1}$ contact impurity-scattering potential, however, we assume a Yukawa-type potential (see below) of finite range. Our purpose is to examine the effect of this finite range on the transport properties. We perform our calculations for $d=2$.

We choose a model scattering potential

$$
V(r)=\alpha \frac{k_{F}}{2 \pi} \frac{\exp \left(-\gamma k_{F} r\right)}{r},
$$

where $\alpha$ is a constant to be specified later on, $k_{F}$ is the Fermi momentum, and $\left(\gamma k_{F}\right)^{-1}$ is the range of the potential. The Fourier transform of $V(r)$ in $2 \mathrm{D}$ is

$$
V(q)=\frac{\alpha}{\sqrt{\gamma^{2}+q^{2} / k_{F}^{2}}} .
$$

The quantity of interest in the subsequent scattering process is

$$
\begin{aligned}
& n_{I} V^{2}(q)=\frac{C_{0}}{\gamma^{2}+q^{2} / k_{F}^{2}}, \\
& C_{0}=n_{I} \alpha^{2} .
\end{aligned}
$$

$n_{I}$ is the number of impurities per unit surface. Since all the electronic momenta are of order $k_{F}$, we have $|\mathbf{k}| \sim\left|\mathbf{k}^{\prime}\right| \sim k_{F}$ :

$$
\begin{aligned}
& n_{I} V^{2}\left(\mathbf{k}-\mathbf{k}^{\prime}\right) \simeq \frac{C_{0}}{2} \frac{1}{\cosh \varphi-\mathbf{k} \cdot \mathbf{k}^{\prime} / k_{F}^{2}}, \\
& \cosh \varphi=\left(\gamma^{2} / 2\right)+1
\end{aligned}
$$

We now discuss various choices for $\alpha$ and $\gamma$.

(a) $\alpha \equiv \gamma \rightarrow \infty$. In this case $V(r)$ is normalized to 1 and
$V(q)$ is a constant. One thus recovers the contact potential and the usual results ${ }^{1}$ for the conductivity, as will be checked in the following.

(b) $\alpha \equiv \sqrt{2}, \gamma \neq 0$. ( $\sqrt{2}$ is chosen for convenience, but it can be any finite constant). In the limit $\gamma=0, V(r)$ is proportional to the pure Coulomb potential, which is known to be pathological: ${ }^{2} V(r)$ cannot be normalized, since the differential-scattering cross section diverges, due to this potential (the well-known Rutherford formula ${ }^{2}$ ), and the Born approximation, which will be used in the present paper in the standard manner, ${ }^{1}$ is questionable. Furthermore, an infinite range is not compatible with our independent-impurities hypothesis.

Therefore, in the following we confine ourselves to finite values $\gamma \neq 0$. However, we will be interested, in particular, in small values of $\gamma, 0<\gamma<1$, corresponding to an "almost long-range" potential. As is well known, ${ }^{3}$ only $\gamma=0$ yields a true long-range potential. Nevertheless, when $0<\gamma<1$, the potential extends rather far and it is the physics of this case which motivated our work.

When the localization transition is approached, the electrons slow down and it takes longer for the screening to become effective. Therefore, it is reasonable to assume that the interaction between a conduction electron and the extra charge introduced by the impurity, i.e., the scattering potential that the electrons feel, tends to become more of an "almost pure Coulomb type," at least on a short time scale. Our model potential (1), with $\gamma<1$, is intended to mimic precisely this physical situation. If these ideas are correct, one should find that a finite range for the impurity potential reinforces the localization. We will show explicitly in the present paper that this is indeed the case.

(c) $\alpha \equiv \gamma \neq 0$. This case is the same as (b), except that here, the potential is normalized to 1 . One gets an almost pure Coulomb potential but with a very small amplitude. The restrictions pointed out for case (b), when $\gamma \sim 0$, apply here as well.

In the following, we concentrate on cases (a) and (b). Our paper is organized as follows. In Sec. II, we compute, within the Born approximation, the electron elastic lifetime due to the scattering potential (2). We also compute $\Gamma_{c}^{(n+1)}\left(\mathbf{k}, \mathbf{k}^{\prime}\right)$, the $(n+1)$ th order of the Cooperon $\Gamma_{c}\left(\mathbf{k}, \mathbf{k}^{\prime}\right)$, i.e., the ladder with $n$ single impurity scatterings in the particle-particle channel. $\Gamma_{c}\left(\mathbf{k}, \mathbf{k}^{\prime}\right)$ itself is then given by

$$
\Gamma_{c}\left(\mathbf{k}, \mathbf{k}^{\prime}\right)=\sum_{n=1}^{\infty} \Gamma^{(n+1)}\left(\mathbf{k}, \mathbf{k}^{\prime}\right),
$$


with the appropriate fermionic Matsubara frequencies implicitly included. In Sec. III, we collect all the diagrams contributing to the electrical conductivity $\sigma$, in the weakly localized regime, and we discuss the resulting formula for $\sigma$. In Sec. IV, we conclude and correlate our work with previous works dealing with what is usually called "correlated disorder."

\section{CALCULATION OF THE COOPERON $\boldsymbol{\Gamma}_{c}$}

\section{A. The relaxation time $\tau$}

In $2 \mathrm{D}$ the elastic scattering relaxation time $\tau$ is given, in the Born approximation, by the usual ${ }^{4}$ diagram of Fig. 1. Using (3) and (4),

$$
\frac{1}{\tau}=n_{I} \int_{0}^{2 \pi} \frac{d \theta^{\prime}}{2 \pi} V^{2}\left(\mathbf{k}-\mathbf{k}^{\prime}\right)=\frac{C_{0}}{2 \sinh \varphi}
$$

(here $\theta^{\prime}$ is the angle between $\mathbf{k}$ and $\mathbf{k}^{\prime}$ ) and $|\mathbf{k}|=\left|\mathbf{k}^{\prime}\right|=k_{F}$. Retaining only the diagram of Fig. 1 for the electron self-energy correction preserves the Ward identity ${ }^{5}$ that guarantees the conservation of the total number of particles.

For the choices of $\alpha$ mentioned in the Introduction, we obtain the following.

(a) $\tau^{-1}=n_{I}[\cosh \varphi-1] / \sinh \varphi \rightarrow n_{I}$, when $\varphi \rightarrow \infty$. One recovers the usual formula for the contact scattering potential.

(b) $\tau^{-1}=n_{I} / \sinh \varphi$. In the limit $\varphi \rightarrow 0, \tau^{-1}$ diverges. This last result corresponds to the Rutherford formula mentioned in the Introduction for a pure Coulomb potential.

\section{B. The calculation of $\Gamma_{c}^{(n+1)}$}

We now compute the $(n+1)$ th order of the Cooperon, $\Gamma_{c}^{(n+1)}$ shown in Fig. 2. We can write it as (using $\mathbf{p}$ for $\mathbf{k}_{n}$ in the figure)

$$
\begin{aligned}
& \Gamma_{c}^{(n+1)}\left(\mathbf{k}, \mathbf{k}^{\prime}, \mathbf{Q} ; \widetilde{\omega}_{n+v}, \widetilde{\omega}_{n}\right) \\
& =\int \frac{d \mathbf{p}}{(2 \pi)^{2}} \Gamma_{c}^{(n)}\left(\mathbf{k}, \mathbf{p}, \mathbf{Q} ; \widetilde{\omega}_{n+v}, \widetilde{\omega}_{n}\right) \\
& \quad \times G\left(\mathbf{p}, \widetilde{\omega}_{n+v}\right) G\left(\mathbf{Q}-\mathbf{p}, \widetilde{\omega}_{n}\right) n_{I} V^{2}\left(\left|\mathbf{p}-\mathbf{k}^{\prime}\right|\right)
\end{aligned}
$$

$G\left(\mathrm{p}, \widetilde{\omega}_{n}\right)$ is the one-particle Green's function for momentum $\mathbf{p}$ and Matsubara frequency $\widetilde{\omega}_{n}$. It contains the elastic relaxation time $\tau$ due to impurity scattering through $\widetilde{\omega}_{n}=\omega_{n}+(2 \tau)^{-1} \operatorname{sgn} \omega_{n}$, with $\omega_{n}=2 \pi T\left(n+\frac{1}{2}\right)$, with $T$ being the temperature. $\tau$ is given by (6). In atomic units

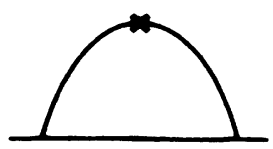

FIG. 1. The usual diagram (Ref. 4) for computing the lifetime $\tau$ [formula (6)]. The straight line is the electron line; the rounded line with a cross denotes the impurity scattering.

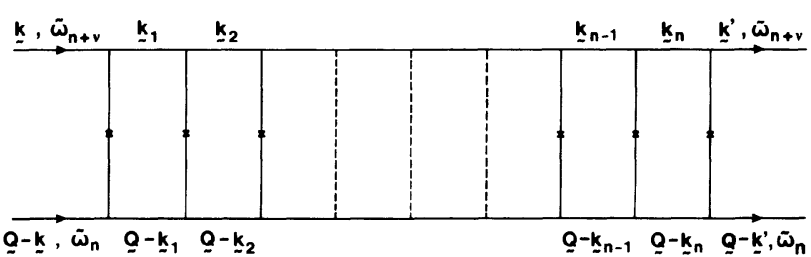

FIG. 2. The $(n+1)$ th order of the Cooperon $\Gamma_{c}^{(n+1)}\left(k, k^{\prime} ; \widetilde{\omega}_{n+v}, \widetilde{\omega}_{n}\right)$ given by formula (12). The upper and lower straight lines are the two electron lines renormalized by impurity scattering according to Fig. 1 . The vertical lines with crosses denote impurity scattering. $\boldsymbol{Q}$ is the overall momentum of the Cooperon. $\omega_{v}$ is the external frequency coming into and going out of the conductivity diagrams in Fig. 3, which will eventually be taken in the limit $\omega_{v} \rightarrow 0 ; \widetilde{\omega}_{n+v}, \widetilde{\omega}_{n}$ are the Matsubara frequencies of the two electron lines and the various $k$ 's and $(\boldsymbol{Q}-\boldsymbol{k})$ 's, their respective momenta.

(a.u.), $G$ is given by

$$
\begin{aligned}
& G\left(\mathbf{p}, \widetilde{\omega}_{n}\right)=\left(i \widetilde{\omega}_{n}-\xi_{p}\right)^{-1}, \\
& \xi_{p}=\left(p^{2}-k_{F}^{2}\right) / 2 .
\end{aligned}
$$

$n_{I} V^{2}\left(\left|\mathbf{p}-\mathbf{k}^{\prime}\right|\right)$ is given by (3), with $q \equiv\left|p-\mathbf{k}^{\prime}\right|$, or by (4) as

$$
n_{I} V^{2}\left(\left|\mathbf{p}-\mathbf{k}^{\prime}\right|\right)=\frac{C_{0}}{2} \frac{1}{\cosh \varphi-\cos \left(\theta_{k},-\theta_{p}\right)},
$$

where $\theta_{k^{\prime}}\left(\theta_{p}\right)$ is the angle between $\mathbf{k}^{\prime}(\mathbf{p})$ and the reference axis. Usually, for a contact potential [case (a) in the Introduction], $n_{I} V^{2}\left(\left|\mathbf{p}-\mathbf{k}^{\prime}\right|\right)$ reduces to a constant, $n_{I}$. Then the right-hand side of (7) is independent of $\mathbf{k}^{\prime}$ and so is the left-hand side. Since $\Gamma_{c}^{(n+1)}\left(\mathbf{k}, \mathbf{k}^{\prime}\right)$ is independent of $\mathbf{k}^{\prime}, \Gamma_{c}^{(n)}(\mathbf{k}, \mathbf{p})$ is independent of $\mathbf{p}$ and can be pulled out from the integral, which reduces to the integral over $p$ of the product of the two Green's functions. Then one recovers the geometric series ${ }^{1}$ for $\Gamma_{c}$ leading to the usual diffusive pole $\left(\omega_{v}+D\left|\mathbf{k}-\mathbf{k}^{\prime}\right|^{2}\right)^{-1}$, with $D$ the diffusion constant given by $D=k_{F}^{2} \tau / 2$ in a.u.. In the general case, however, when $V^{2}\left(\left|\mathbf{p}-\mathbf{k}^{\prime}\right|\right)$ depends explicitly on the angles $\theta_{p}$ and $\theta_{k^{\prime}}$ the right-hand side of (7) is not separable. Therefore, before computing $\Gamma_{c}$, we must study $\Gamma_{c}^{(n+1)}$. (Note that $n$ starts at $n=1$ because the $n=0$ contribution is already taken into account in the diffuson of the Drude term of the conductivity. This will become explicit in Sec. III). $\mathbf{Q}$ is the momentum of the Cooperon contributing to the diffusive pole when $|\mathbf{Q}| \rightarrow 0$ or, more precisely, when $k_{F} Q \tau<1$ (and $\omega_{v} \tau<1$ ). In the following, we expand the Green's function $G(\mathbf{Q}-\mathbf{p})$ in (7) as follows:

$$
\begin{aligned}
G\left(\mathbf{Q}-\mathbf{p}, \widetilde{\omega}_{n}\right) \approx & G\left(\mathbf{p}, \widetilde{\omega}_{n}\right)-\mathbf{p} \cdot \mathbf{Q} G^{2}\left(p, \widetilde{\omega}_{n}\right) \\
& +(\mathbf{p} \cdot \mathbf{Q})^{2} G^{3}\left(\mathbf{p}, \widetilde{\omega}_{n}\right)+\cdots .
\end{aligned}
$$

Using (10), we get 


$$
\begin{aligned}
\int \frac{d^{2} \mathbf{p}}{(2 \pi)^{2}} \boldsymbol{G}\left(p, \widetilde{\omega}_{n+v}\right) \boldsymbol{G}\left(\mathbf{Q}-\mathbf{p}, \widetilde{\omega}_{n}\right) & =\int \frac{d \theta_{p}}{2 \pi} \int \frac{d \xi_{p}}{2 \pi} G\left(p, \widetilde{\omega}_{n}\right) \boldsymbol{G}\left(\mathbf{Q}-\mathbf{p}, \widetilde{\omega}_{n}\right) \\
& \approx \frac{1}{\omega_{v}+\frac{1}{\tau}} \int \frac{d \theta_{p}}{2 \pi}\left[1-i k_{F} Q \tau \cos \left(\theta_{Q}-\theta_{p}\right)-k_{F}^{2} Q^{2} \tau^{2} \cos ^{2}\left(\theta_{Q}-\theta_{p}\right)\right]
\end{aligned}
$$

We then find for $\Gamma_{c}^{(n+1)}$

$$
\begin{array}{r}
\Gamma_{c}^{(n+1)}\left(\mathbf{k}, \mathbf{k}^{\prime}, \mathbf{Q} ; \widetilde{\omega}_{n+\imath} \widetilde{\omega}_{n}\right) \\
=\left(\frac{C_{0}}{2}\right)^{n+1}\left(\frac{1}{\left.\omega_{v}+\frac{1}{\tau}\right)^{n} \int \cdots \int} \frac{d \theta_{1}}{2 \pi} \frac{d \theta_{2}}{2 \pi} \cdots \frac{d \theta_{n}}{2 \pi} \frac{1}{\cosh \varphi-\cos \left(\theta_{1}-\theta\right)} \frac{1}{\cosh \varphi-\cos \left(\theta_{2}-\theta_{1}\right)}\right. \\
\times \\
\times\left\{\frac{1}{\cosh \varphi-\cos \left(\theta_{n}-\theta_{n-1}\right)} \frac{1}{\cosh \varphi-\cos \left(\theta^{\prime}-\theta_{n}\right)}\right. \\
\times\left\{1-i k_{F} Q T \sum_{i=1}^{n} \cos \left(\theta_{Q}-\theta_{i}\right)\right. \\
\left.-k_{F}^{2} Q^{2} T^{2} \sum_{\substack{i, j=1 \\
i<j}}^{n} \cos \left(\theta_{Q}-\theta_{i}\right) \cos \left(\theta_{Q}-\theta_{j}\right)-k_{F}^{2} Q^{2} T^{2} \sum_{i=1}^{n} \cos ^{2}\left(\theta_{Q}-\theta_{i}\right)\right\} .
\end{array}
$$

$\theta_{i}, \theta$, and $\theta^{\prime}$ stand for $\theta_{\mathbf{k}_{i}}, \theta_{k}$, and $\theta_{k}$, respectively. The integrals are cumbersome but tractable and they are calculated in Appendix A.1 with the result

$$
\begin{aligned}
\Gamma_{c}^{(n+1)}\left(\mathbf{k}, \mathbf{k}^{\prime}, \mathbf{Q} ; \widetilde{\omega}_{n+v}, \widetilde{\omega}_{n}\right)= & \frac{1}{\tau}\left[\frac{\tau^{-1}}{\omega_{v}+\tau^{-1}}\right]^{n} \frac{1}{\cosh [(n+1) \varphi]-\cos \theta^{\prime}} \\
\times & {\left[\mid 1-\frac{n k_{F}^{2} Q^{2} \tau^{2}}{2}\right) \sinh [(n+1) \varphi] } \\
& -\frac{k_{F}^{2} Q^{2} \tau^{2}}{4} \mid n \frac{\cosh \left[\left(n+\frac{1}{2}\right) \varphi\right]-\cos \theta^{\prime} \cosh (\varphi / 2)}{\sinh (\varphi / 2)} \\
& \left.\left.+\frac{\sinh (n \varphi / 2)}{\sinh ^{2}(\varphi / 2)}\left\{\cosh \left[\left(\frac{n}{2}+1\right) \varphi\right]-\cos \theta^{\prime} \cosh \mid \frac{n \varphi}{2}\right]\right\}\right] \\
& \left.-\frac{k_{F}^{2} Q^{2} \tau^{2}}{4}\left[\cos 2 \theta_{Q} S_{0}(n)+\cos \left(2 \theta_{Q}-\theta^{\prime}\right) S_{1}(n)+\cos \left(2 \theta_{Q}-2 \theta^{\prime}\right) S_{2}(n)\right]\right\rfloor,
\end{aligned}
$$

where we used Eq. (6). In (13) we have assumed the reference axis to be along $\mathbf{k}$, so that $\theta=0$. The coefficients $S_{i}$ for $i=0,1,2$ are complicated expressions and are given by

$$
\begin{aligned}
& S_{0}(n)=\frac{1}{\left(e^{\varphi}-1\right)}\left(2 \frac{\sinh \left[\left(n+\frac{1}{2}\right) \varphi\right] \sinh (n \varphi / 2)}{\sinh (\varphi / 2)}+\frac{\sinh (n \varphi)}{\sinh (\varphi)}\left(e^{\varphi}-2\right)+n e^{-(n+1) \varphi}\right), \\
& S_{1}(n)=\frac{1}{\left(e^{\varphi}-1\right)^{2}}\left[2 \cosh [(n+1) \varphi]\left[1-e^{-n \varphi}-n\left(e^{\varphi}-1\right) e^{-(n+1) \varphi}\right]\right. \\
& \left.-e^{-n \varphi} 2 \sinh (n \varphi) \tanh \left[\frac{\varphi}{2}\right]+e^{-n \varphi}\left(1-e^{-n \varphi}\right)\right], \\
& S_{2}(n)=\frac{1}{\left(e^{\varphi}-1\right)}\left[e^{\varphi} \frac{\sinh (n \varphi)}{\sinh (\varphi)}-\operatorname{coth}\left(\frac{\varphi}{2}\right)\left(1-e^{-n \varphi}\right)+n e^{-(n+1) \varphi}\right]
\end{aligned}
$$


We notice in Eq. (13) a surprising term linear in $Q$ for arbitrary $\theta^{\prime}$ (it vanishes only for $\theta^{\prime}=\pi$ ) but this term is, in fact, irrelevant and it can be neglected, as we show in the following discussion.

Note that we could have simplified more drastically formula (12), taking advantage of the following fact. In the subsequent calculation of the conductivity, we will have to integrate over $\mathbf{Q}$; in particular, we will need $\int_{0}^{2 \pi} d \theta_{\mathbf{Q}} /(2 \pi)$. Therefore, immediately integrating (12) over $d \theta_{\mathrm{Q}}$ would amount to replacing the expression in $\{\cdots\}$ in (12) by

$$
\left\{1-\frac{k_{F}^{2} Q^{2} \tau^{2}}{2} \sum_{\substack{i, j=1 \\ i<j}}^{n} \cos \left(\theta_{i}-\theta_{j}\right)-n \frac{k_{F}^{2} Q^{2} \tau^{2}}{2}\right\}
$$

This, in turn, would result in suppressing in (13) the imaginary linear term in $Q$ and the following $Q^{2}$ term. The evaluation of Eq. (12) with this last form is given in Appendix A.2.

\section{The calculation of $\Gamma_{c}$}

We now compute $\Gamma_{c}$ in Eq. (5), together with Eqs. (13) and (14). Performing the sums over $n$ is very difficult, so we will examine specifically the two cases (a) and (b) of the Introduction.

$$
\text { (a) } \alpha \equiv \gamma \rightarrow \infty
$$

Since $n \geq 1$, and $\varphi \rightarrow \infty$, then $n \varphi \rightarrow \infty$ and the dominant terms in Eq. (13) are

$$
\begin{aligned}
\Gamma_{c}^{(n+1)}\left(\mathbf{k}, \mathbf{k}^{\prime}, \mathbf{Q} ; \widetilde{\omega}_{n+v}, \widetilde{\omega}_{n}\right) \approx \frac{1}{\tau}\left[\frac{\tau^{-1}}{\omega_{v}+\tau^{-1}}\right]^{n}\{ & {\left[1-n \frac{k_{F}^{2} Q^{2} \tau^{2}}{2}\right] } \\
+ & -\frac{k_{F}^{2} Q^{2} \tau^{2}}{2} \frac{n}{\left(e^{\varphi}-1\right)} \\
& +\frac{k_{F}^{2} Q^{2} \tau^{2}}{8 \sinh ^{2}(\varphi / 2)}\left[1-\cos \theta^{\prime} e^{-\varphi}-\cos \left(2 \theta_{Q}\right) \frac{2 e^{\varphi}-1}{\left(e^{\varphi}+1\right)}\right. \\
& \left.\left.-\cos \left(2 \theta_{Q}-\theta^{\prime}\right) e^{-\varphi}-\cos \left(2 \theta_{Q}-\theta^{\prime}\right) \frac{1}{\left(e^{\varphi}-1\right)}\right]\right\},
\end{aligned}
$$

which reduces to

$$
\Gamma_{c}^{(n+1)}\left(\mathbf{k}, \mathbf{k}^{\prime}, \mathbf{Q} ; \widetilde{\omega}_{n+v}, \widetilde{\omega}_{n}\right) \approx \frac{1}{\tau}\left[\frac{\tau^{-1}}{\omega_{v}+\tau^{-1}}\right]^{n}\left[1-n \frac{k_{F}^{2} Q^{2} \tau^{2}}{2}+\epsilon\right] \approx \frac{1}{\tau}\left[\frac{1}{1+\omega_{v} \tau}\right]^{n}\left[1-n \frac{k_{F}^{2} Q^{2} \tau^{2}}{2}\right]^{n}(1+\epsilon)
$$

where we indicated by $\epsilon$ the sums of terms proportional to $Q$ and $Q^{2}$ that are not multiplied by $n$. Then,

$$
\begin{aligned}
\Gamma_{c}^{(n+1)}\left(\mathbf{k}, \mathbf{k}^{\prime}, \mathbf{Q} ; \widetilde{\omega}_{n+v}, \widetilde{\omega}_{n}\right) & \approx(1+\epsilon) \frac{1}{\tau} \sum_{n=1}^{\infty}\left[\frac{1}{1+\omega_{v} \tau}\left[1-\frac{k_{F}^{2} Q^{2} \tau^{2}}{2}\right]\right]^{n} \\
& \approx \frac{1}{\tau^{2}} \frac{1}{\omega_{v}+D Q^{2}}, \tau^{-1}=n_{I}, \quad D=\frac{k_{F}^{2} \tau}{2}
\end{aligned}
$$

where $\epsilon(\ll 1)$ gives an uninteresting correction to unity in the numerator and it can be dropped. We thus recover the usual pole ${ }^{1}$ in the diffusive regime for the Cooperon $\Gamma_{c}$.

$$
\text { (b) } \alpha=\sqrt{2} \text {, arbitrary } \gamma
$$

In the general case, we separate in (5)

$$
\sum_{n=1}^{\infty}=\sum_{n=1}^{n_{0}}+\sum_{n=n_{0}+1}^{\infty}
$$

$n_{0}$ is chosen such that, for a given value of $\varphi$ (i.e., for a given value of the potential range),

$$
n_{0} \varphi=1
$$

We recall that for reasons explained in the Introduction, we exclude the value $\gamma=0$, i.e., the value $\varphi=0$. Thus, $\varphi$ is always finite and the $\sinh (\varphi / 25)$ and $\left(e^{\varphi}-1\right)$ appearing in the denominators of (13) and (14) are also finite quantities. Under these conditions, the first sum on the right-hand side of $(18)$ is a finite sum of finite terms, which thus cannot yield a diffusive pole for $\Gamma_{c}$; we ignore it in the following. In the second term of the right-hand side of (18), we put

$$
n=n_{0}+1+n^{\prime}
$$


Moreover, since $n \varphi>1$, we can replace the hyperbolic functions by their expansions for large arguments.

According to the remark made after Eq. (17), we can drop in (13) the terms in $Q^{2}$ that are not multiplied by $n$ for small values of $Q$. This leaves us with only the first two $Q^{2}$ terms. Then, $n \varphi>1$ and, in the case of later interest, where $\mathbf{k}^{\prime}=\boldsymbol{Q}-\mathbf{k} \approx-\mathbf{k},\left(\cos \theta^{\prime}=-1\right)$, we get for (13)

$\Gamma_{c}^{(n+1)}\left(\mathbf{k},-\mathbf{k}, \mathbf{Q} ; \widetilde{\omega}_{n+v}, \widetilde{\omega}_{n}\right) \approx \tau^{-1}\left[\frac{\tau^{-1}}{\omega_{v}+\tau^{-1}}\right]^{n}\left[1-n \frac{k_{F}^{2} Q^{2} \tau^{2}}{2} \frac{e^{\varphi}}{e^{\varphi}-1}\right] \approx \tau^{-1}\left[\frac{\tau^{-1}}{\omega_{v}+\tau^{-1}}\left[1-\frac{k_{F}^{2} Q^{2} \tau^{2}}{2} \frac{e^{\varphi}}{e^{\varphi}-1}\right]\right]^{n}$.

Then $\Gamma_{C}$ follows straightforwardly as

$\Gamma_{c}\left(\mathbf{k},-\mathbf{k}, \mathbf{Q} ; \widetilde{\omega}_{n+v}, \widetilde{\omega}_{n}\right) \approx \sum_{n^{\prime}=0}^{\infty} \tau^{-1}\left[\frac{\tau^{-1}}{\omega_{v}+\tau^{-1}}\left[1-\frac{k_{F}^{2} Q^{2} \tau^{2}}{2} \frac{e^{\varphi}}{e^{\varphi}-1}\right]\right]^{n^{\prime}} \approx \frac{1}{\tau^{2}} \frac{1}{\omega_{v}+\left(k_{F}^{2} Q^{2} / 2\right) \frac{\tau e^{\varphi}}{e^{\varphi}-1}}, \frac{1}{\tau}=\frac{n_{I}}{\sinh \varphi}$

Therefore, we find that, as usual, ${ }^{1} \Gamma_{C}$ has a diffusive pole (without any constant term in the denominator). This follows from the Ward identity insuring the conservation of the total number of particles as noted after Eq. (6).

As will become clear in the following, the term proportional to $Q^{2}$ in the denominator of (22) involves the transport time $\tau_{\mathrm{tr}}=\tau e^{\varphi} /\left(e^{\varphi}-1\right)$, so that $\Gamma_{C}$ may be written in terms of the "transport diffusion constant" $D_{\mathrm{tr}}$ as

$$
\begin{aligned}
& \Gamma_{C} \approx \frac{1}{\tau^{2}} \frac{1}{\omega_{v}+D_{\mathrm{tr}} Q^{2}}, \\
& D_{\mathrm{tr}} \approx \frac{k_{F}^{2} \tau_{\mathrm{tr}}}{2},
\end{aligned}
$$

where $D_{\mathrm{tr}}$ is, in general, different from $k_{F}^{2} \tau / 2$.

\section{CALCUlation OF THE ELECTRICAL CONDUCTIVITY $\sigma$}

First, we have to collect all the diagrams contributing to $\sigma$. Due to the fact that the scattering potential, in Fourier space, depends on the momentum transfer, many more diagrams contribute, compared to the case of contact-scattering potential. We have examined all the topologically possible diagrams. We have found that the only relevant ones, to first order in $\left(\epsilon_{F} \tau\right)^{-1}$, are those shown in Fig. 3. For the contact-potential case, where the scattering potential does not depend on the momentum transfer, two only diagrams matter: the ones labeled (a), yielding the usual ${ }^{1}$ Drude value, and (c), the "multiply crossed" diagram ${ }^{1}$ yielding the localization correction. For comparison, we recall the result ${ }^{1}$ for the contact potential due to diagrams (a) and (c):

$$
\sigma_{(\mathrm{a})+(\mathrm{c})}=\frac{k_{F}^{2}}{2 \pi} \tau-\frac{1}{\pi^{2}} \ln \left(\frac{L}{k_{F} \tau}\right), \quad \tau^{-1}=n_{I}
$$

where $L$ is the linear dimension of the system.

Instead, for $\alpha=\sqrt{2}$ and $\gamma$ finite, we have to take into account all the other diagrams of Fig. 3. The algebra is straightforward; we give some details in Appendix B. Denoting by $\sigma_{(a)}$, etc., the contribution to $\sigma$ of diagrams (a), etc., of Fig. 3, we get

$$
\sigma_{(\mathrm{a})}=\frac{k_{F}^{2}}{2 \pi} \tau, \quad \tau^{-1}=n_{I} / \sinh \varphi
$$

In Fig. 3, the diffuson $\Gamma_{d}$ (square box) is the infinite impurity-scattering ladder in the particle-hole channel. Its analytic expression can be obtained from $\Gamma_{C}$ with
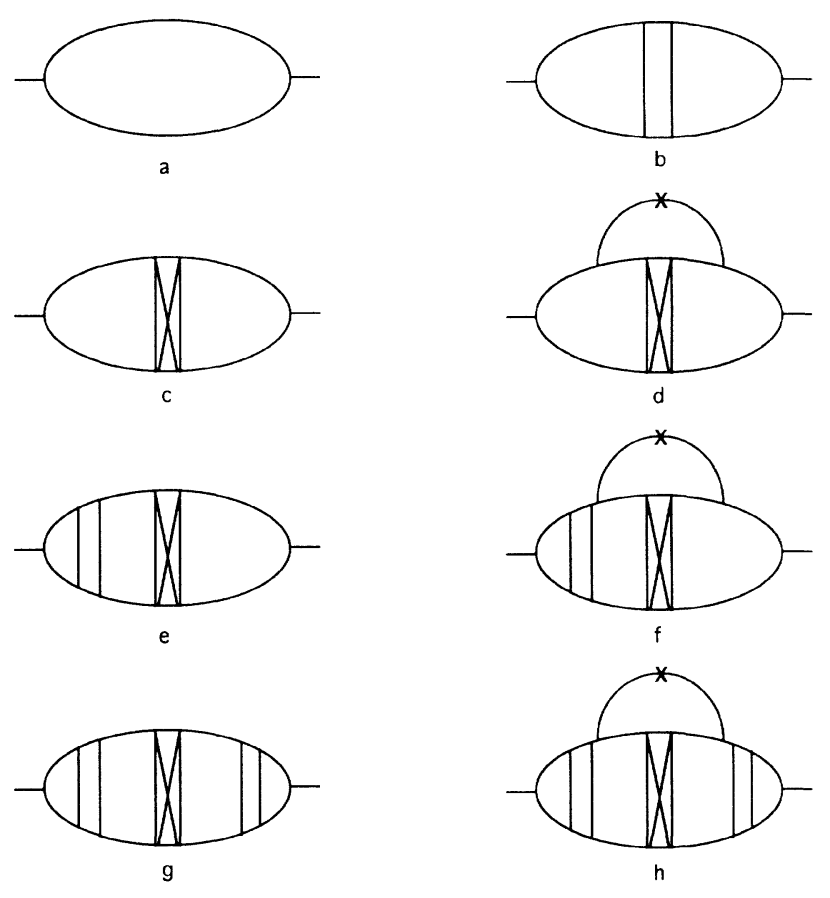

FIG. 3. The relevant diagrams in the present problem, for the conductivity $\sigma$, to first order in $\left(\epsilon_{F} \tau\right)^{-1}$ : the square box denotes the diffuson $\Gamma_{d}$ [(formula (26)] and the one with diagonals denotes the multiply crossed ladder, i.e., the Cooperon $\Gamma_{c}$ given by formula (5) with (13) and (14). The lines with crosses are impurity-scattering lines. The other lines are electron lines renormalized by impurity scattering. By symmetry, there exist two diagrams of the type (d), two of the type (e), two (h), and four (f). Diagrams (d), (b), and (h) involve independent momenta $k$ and $\boldsymbol{k}^{\prime}$; instead, diagrams (c), (e), and (g) involve $\boldsymbol{k}^{\prime} \equiv \boldsymbol{Q}-\boldsymbol{k} \approx-\boldsymbol{k}$. Each extra single-impurity line introduces a factor of the form (4). 
$Q=0$, and with the summation in (5) including the $n=0$ term. Finally,

$$
\begin{aligned}
\Gamma_{d}^{(n+1)}\left(\mathbf{k}, \mathbf{k}^{\prime}, \mathbf{Q}, \widetilde{\omega}_{n+v}, \widetilde{\omega}_{n}\right)=\sum_{n=0}^{\infty} & \frac{1}{\tau}\left[\frac{\tau^{-1}}{\omega_{v}+\tau^{-1}}\right]^{n} \\
& \times \frac{\sinh (n+1) \varphi}{\cosh (n+1) \varphi-\cos \theta^{\prime}} .
\end{aligned}
$$

Whenever $\mathbf{k}^{\prime}$ is independent of $\mathbf{k}$, we found it easier to compute the corresponding conductivity diagrams, involving either the diffuson or the Cooperon, with the $(n+1)$ th-order term, then integrate over $\mathbf{k}^{\prime}$ and finally sum over $n$. We thus found

$$
\sigma_{(\mathrm{b})}=\sigma_{(\mathrm{a})} \frac{1}{e^{\varphi}-1},
$$

with $\sigma_{(\mathrm{a})}$ given by (25).

The diagrams containing Cooperons yield

$$
\begin{aligned}
\sigma_{(\mathrm{c})} & =-\frac{1}{\pi} \int \frac{Q d Q}{2 \pi}\left\langle\Gamma_{c}(\mathbf{k},-\mathbf{k})\right\rangle_{\omega_{v}=0} k_{F}^{2} \tau^{3} \\
& =-\frac{1}{\pi^{2}} \frac{e^{\varphi}-1}{e^{\varphi}} \ln \left[\frac{L}{k_{F} \tau}\right], \quad \tau^{-1}=\frac{n_{I}}{\sinh \varphi}
\end{aligned}
$$

with

$$
\begin{aligned}
& \left\langle\Gamma_{c}(\mathbf{k},-\mathbf{k})\right\rangle_{\omega_{v}=0} \\
& =\lim _{\omega_{v}=0} \int \frac{d \theta_{Q}}{2 \pi} \Gamma_{c}\left(\mathbf{k},-\mathbf{k}, \mathbf{Q} ; \widetilde{\omega}_{n+v}, \widetilde{\omega}_{n}\right) \\
& \sigma_{(\mathrm{e})}=\sigma_{(\mathrm{c})} 2 \frac{1}{e^{\varphi}-1}, \\
& \sigma_{(\mathrm{g})}=\sigma_{(\mathrm{c})} \frac{1}{\left(e^{\varphi}-1\right)^{2}}
\end{aligned}
$$

On the other hand,

$$
\begin{aligned}
\sigma_{(\mathrm{d})}=-\frac{1}{\pi} \sum_{n} \int \frac{Q d Q}{2 \pi} \int \frac{d \theta^{\prime}}{2 \pi}\left\langle\Gamma_{c}^{(n+1)}(\mathbf{k},-\mathbf{k})\right\rangle_{\omega_{v}=0} \\
\quad \times k_{F}^{2} \tau^{3} \frac{\cos \theta^{\prime}}{\cosh \varphi+\cos \theta^{\prime}} \sinh \varphi \\
=-\sigma_{(\mathrm{c})} e^{-\varphi} \\
\sigma_{(\mathrm{f})}=\sigma_{(\mathrm{d})} 2 \frac{1}{e^{\varphi}-1} \\
\sigma_{(\mathrm{h})}=\sigma_{(\mathrm{d})} \frac{1}{\left(e^{\varphi}-1\right)^{2}}
\end{aligned}
$$

Therefore

$$
\begin{aligned}
& \sigma=\sigma_{\text {Drude }}+\sigma_{\text {loc }}, \frac{1}{\tau}=\frac{n_{I}}{\sinh \varphi}, \\
& \sigma_{\text {Drude }}=\sigma_{(\mathrm{a})}+\sigma_{(\mathrm{b})} \\
& =\frac{k_{F}^{2} \tau}{2 \pi}\left[1+\frac{1}{e^{\varphi}-1}\right] \\
& =\frac{k_{F}^{2}}{2 \pi} \tau_{\mathrm{tr}},
\end{aligned}
$$

with $\tau_{\mathrm{tr}}$ defined before Eq. (23). Expressed in terms of $\varphi$ only, using the explicit expression for $\tau$ recalled above in (34), we get

$$
\begin{aligned}
\sigma_{\text {Drude }}= & \frac{k_{F}^{2}}{2 \pi n_{I}} \frac{e^{\varphi}+1}{2} \\
\sigma_{\text {loc }}= & \sigma_{(\mathrm{c})}+\cdots+\sigma_{(\mathrm{h})} \\
= & -\left(1-e^{-\varphi}\right)\left[\frac{e^{\varphi}}{e^{\varphi}-1}\right]^{2}\left[\frac{e^{\varphi}-1}{e^{\varphi}}\right] \frac{1}{\pi^{2}} \\
& \times \ln \left[\frac{L}{k_{F} \tau}\right]=-\frac{1}{\pi^{2}} \ln \left[\frac{L}{k_{F} \tau}\right] .
\end{aligned}
$$

Clearly, when the potential range increases ( $\varphi$ decreases), $\sigma_{\text {Drude }}$ decreases. On the other hand, the coefficient of the ln in $\sigma_{\text {loc }}$ remains the same as in the case of the contact potential. The effect of quantum localization is nevertheless reinforced through the dependence of $\tau$ [under the $\ln$ in (37)] on the range of the potential. As a consequence, the total conductivity $\sigma$ in (34) decreases.

\section{CONCLUSION}

The result of the preceding section is consistent with our initial idea that increasing the range of the impurity potential reinforces the tendency of the electrons to localize. In order to handle the mathematical complexities in deriving our result, we had to resort to a number of constraints; the perturbation expansion to first order in $\left(\epsilon_{F} \tau\right)^{-1}$, the Born approximation, and also the independent impurity hypothesis prevent us from examining the direct neighborhood of the Anderson transition. If one estimates, using Ref. 2, the lowest $\varphi$ value allowed for the Born approximation to hold, one finds that $\gamma$ can be quite low $(>0.4)$. On the other hand, concerning the validity of our independent-impurity hypothesis, one must note that the potential range cannot exceed the mean distance between impurities, denoted by $\left(2 r_{0}\right)$. With the density of impurities being $n_{I}$, we have, in $2 \mathrm{D},\left(\pi r_{0}^{2}\right)=n_{I}^{-1}$. Therefore, $\gamma^{-1}$ cannot exceed $\gamma_{0}^{-1}$ such that $\left(\gamma_{0} k_{F}\right)^{-1}=2 r_{0}$. For small $\gamma, \varphi \sim \gamma$, we must have $\varphi>\varphi_{0}$ with $\varphi_{0}=\gamma_{0}$ :

$$
\varphi>\frac{\sqrt{\pi n_{I}}}{2 k_{F}} .
$$

We may still handle small values of $\varphi$ if the impurity density remains weak, according to the above expression which, in turn, puts a limitation on the possible increase of $\tau^{-1}$ :

$$
\frac{1}{\tau}<\left(\frac{n_{I}}{\pi}\right)^{1 / 2} 2 k_{F} .
$$

Despite these constraints, we believe that the present paper shows, at least qualitatively, how a finite-range impurity potential enhances the electronic localization.

Anderson localization with correlated disorder has been discussed in a number of previous studies. Following the work of Weinrib and Halperin ${ }^{7}$ in magnetic systems, Varriale and Theumann ${ }^{8}$ considered the case of 
algebraically decaying correlations. This would correspond to writing, instead of Eq. $(3), V^{2}(q) \approx q^{\sigma}$, for arbitrary $\sigma \neq 2$. It is well known that such correlations belong to the long-range universality class ${ }^{3}$ and a renormalization group calculation performed in Ref. 8 indicated a new transition for $\sigma<0$, although the singular behavior of the conductivity was analyzed to first order in $\epsilon=4-d$ and the Cooperon was not calculated. Hikami and Brézin ${ }^{9}$ investigated the behavior of the conductivity of a two-dimensional system in a strong magnetic field. Instead of Eq. (3), they assumed a Gaussian form for $V^{2}(q)$ corresponding to a potential $V(r)$ regular at the origin. Depending on the parameter values, they found either a zero conductivity or recovered the results of the uncorrelated unitary case. John and Stephen ${ }^{10}$ reexamined the Anderson localization in $d=2+\epsilon$ dimensions within the nonlinear $\sigma$ model with either exponential or power-law (regular at the origin) correlations. Their results were identical with those of the uncorrelated case even for long-range correlations. However, as the authors remarked, due to the method of calculation, it is possible that their model even in the apparently long-range case, corresponds to a small $q$ expansion in (3) with the form $V^{2}(q) \approx a+b q^{2}$. This would explain why they recover the results of the uncorrelated case. Similar results were obtained in Ref. 11 where, along with a zero-range impurity-scattering potential, a weak, but finite, interaction between the impurities was assumed. The weak local ordering of the impurities (due to their mutual interactions) amounted to having again an "effective" $V^{2}(q)$ of the form $\left(a+b q^{2}\right)$.

Our present work differs in several ways from the above-mentioned studies of correlated disorder. Our model scattering potential, given by (1), is the consequence of physical considerations on the nature of the interaction between electrons and impurities. Since we allow the "screening length" proportional to $1 / \gamma$ to be large, we have to retain the full expression (3), and no expansion in terms of the momentum transfer $q$ is possible. The scattering potential, given by (1), is singular at the origin, contrary to all the earlier studied cases in $2 \mathrm{D}$. We have proved that, even under such conditions, the coefficient of the $\ln$ in $\sigma_{\text {loc }}$ remains the same as that for the contact potential. This is consistent with the fact that for any nonzero value of $\gamma$ (no matter how small it is) the system belongs to the short-range universality class. This is true despite the fact that for small $\gamma$, no small- $q$ expansion of (3) is allowed.

In conclusion, then, we have been able to show explicitly that a finite-range scattering potential reinforces the tendency for localization. This is physically quite reasonable. In the contact-potential case, an electron "feels" an impurity only when they are both in contact. Instead, for a finite-range potential, an electron feels the effect of all the impurities within that range around it. This is equivalent to the electrons being scattered effectively by many more impurities. The case of a finite-range impurity potential is then analogous to the case of a contact potential but with a higher concentration of impurities, which explains the enhanced tendency for localization.

\section{ACKNOWLEDGMENTS}

The authors benefited from fruitful discussions with $\mathbf{P}$. Nozières and B. Altshuler. Their comments are greatfully acknowledged. One of us (A.T.) wishes to acknowledge the hospitality and partial support of the Universite de Paris-Sud. She was partially supported by the Conselho National de Desenvolvimento Cientifico e Technologico (CNPq). Two of us (M.T.B.M. and G.F.) were supported by NATO under Grant No. CR 880736. The Laboratoire de Physique des Solides is a Laboratoire associé au Centre National de la Recherche Scientifique.

\section{APPENDIX A}

\section{Calculation of Eq. (12) without integrating over $\theta_{\varphi}$}

We will need the following useful expressions computed straightforwardly by residues in the complex plane:

$$
\begin{aligned}
& J_{1}\left(\varphi_{1}, \varphi_{2}, \Theta^{\prime}\right)=\frac{1}{2 \pi} \int_{0}^{2 \pi} \frac{d \theta}{\left[\cosh \left(\varphi_{1}\right)-\cos \theta\right]\left[\cosh \left(\varphi_{2}\right)-\cos \left(\theta-\Theta^{\prime}\right)\right]} \\
& =\frac{\sinh \left(\varphi_{1}+\varphi_{2}\right)}{\sinh \left(\varphi_{1}\right) \sinh \left(\varphi_{2}\right)} \frac{1}{\cosh \left(\varphi_{1}+\varphi_{2}\right)-\cos \left(\Theta^{\prime}\right)} \\
& J_{2}\left(\varphi_{1}, \varphi_{2}, \Theta^{\prime}, \Theta^{\prime \prime}\right)=\frac{1}{2 \pi} \int_{0}^{2 \pi} \frac{\cos \left(\theta-\Theta^{\prime \prime}\right) d \Theta}{\left[\cosh \left(\varphi_{1}\right)-\cos \theta\right]\left[\cosh \left(\varphi_{2}\right)-\cos \left(\theta-\Theta^{\prime}\right)\right]} \\
& =\frac{\sinh \left(\varphi_{2}\right) \cos \Theta^{\prime \prime}+\sinh \left(\varphi_{1}\right) \cos \left(\Theta^{\prime}-\Theta^{\prime \prime}\right)}{\sinh \left(\varphi_{1}\right) \sinh \left(\varphi_{2}\right)\left[\cosh \left(\varphi_{1}+\varphi_{2}\right)-\cos \left(\Theta^{\prime}\right)\right]} \\
& J_{3}\left(\varphi_{1}, \varphi_{2}, \Theta^{\prime}, \Theta^{\prime \prime}\right)=\frac{1}{2 \pi} \int_{0}^{2 \pi} \frac{\cos ^{2}\left(\theta-\Theta^{\prime \prime}\right) d \theta}{\left[\cosh \left(\varphi_{1}\right)-\cos \theta\right]\left[\cosh \left(\varphi_{2}\right)-\cos \left(\theta-\Theta^{\prime}\right)\right]} \\
& =\frac{1}{2 \sinh \left(\varphi_{1}\right) \cosh \left(\varphi_{2}\right)} \frac{1}{\cosh \left(\varphi_{1}+\varphi_{2}\right)-\cos \Theta} \\
& \times\left[\sinh \left(\varphi_{1}+\varphi_{2}\right)+e^{-\varphi_{1}} \sinh \left(\varphi_{2}\right) \cos 2 \Theta^{\prime \prime}\right. \\
& \left.+e^{-\varphi_{2}} \sinh \left(\varphi_{1}\right) \cos \left(2 \Theta^{\prime \prime}-2 \Theta^{\prime}\right)+2 e^{-\left(\varphi_{1}+\varphi_{2}\right)} \sinh \left(\varphi_{1}\right) \sinh \left(\varphi_{2}\right) \cos \left(2 \Theta^{\prime \prime}-\Theta^{\prime}\right)\right] \text {. }
\end{aligned}
$$


Using Eq. (A1) repeatedly, we can deduce the useful expression

$$
\begin{aligned}
I_{0}\left(l, \theta^{\prime}\right) & =\int_{0}^{2 \pi} \frac{d \theta_{l}}{2 \pi} \frac{1}{\cosh (\varphi)-\cos \left(\theta_{l}-\theta^{\prime}\right)} \int_{0}^{2 \pi} \frac{d \theta_{l-1}}{2 \pi} \frac{1}{\cosh (\varphi)-\cos \left(\theta_{l-1}-\theta_{l}\right)} \cdots \\
& \times \int_{0}^{2 \pi} \frac{d \theta_{1}}{2 \pi} \frac{1}{\cosh (\varphi)-\cos \left(\theta_{2}-\theta_{1}\right)} \frac{1}{\cosh (\varphi)-\cos \left(\theta_{1}\right)} \\
& =\frac{\sinh [(l+1) \varphi]}{[\sinh (\varphi)]^{l+1}} \frac{1}{\cosh [(l+1) \varphi]-\cos \theta^{\prime}}
\end{aligned}
$$

We write Eq. (12) as

$$
\begin{aligned}
& \Gamma_{c}^{(n+1)}\left(\mathbf{k}, \mathbf{k}^{\prime}, \mathbf{Q} ; \widetilde{\omega}_{n+v}, \widetilde{\omega}_{n}\right)=\frac{1}{\tau}\left(\frac{\tau^{-1}}{\tau^{-1}+\omega_{v^{\prime}}}\right]^{n}[\sinh \varphi]^{(n+1)}\left\{I_{0}\left(n, \theta^{\prime}\right)-i k_{F} Q \tau \sum_{j=1}^{n} I_{j}\left(n, \theta^{\prime}\right)\right. \\
&\left.-k_{F}^{2} Q^{2} \tau^{2}\left[\sum_{\substack{i, j=1 \\
i<j}}^{n} I_{i j}(n)+\sum_{i=1}^{n} L_{i}(n)\right]\right\},
\end{aligned}
$$

where $I_{0}\left(n, \theta^{\prime}\right)$ is given by Eq. (A4) with $l \equiv n$ and

$$
\begin{aligned}
& I_{j}\left(n, \theta^{\prime}\right)=\int_{0}^{2 \pi} \frac{d \theta_{n}}{2 \pi} \frac{1}{\cosh (\varphi)-\cos \left(\theta_{n}-\theta^{\prime}\right)} \cdots \int_{0}^{2 \pi} \frac{d \theta_{j}}{2 \pi} \frac{\cos \left(\theta_{Q}-\theta_{j}\right)}{\cosh (\varphi)-\cos \left(\theta_{j}-\theta_{j+1}\right)} \cdots \\
& \times \int \frac{d \theta_{1}}{\cosh (\varphi)-\cos \left(\theta_{1}-\theta_{2}\right)} \frac{1}{\cos (\varphi)-\cos \left(\theta_{1}\right)}, \\
& I_{i j}(n)=\int_{0}^{2 \pi} \frac{d \theta_{n}}{2 \pi} \frac{1}{\cosh (\varphi)-\cos \left(\theta_{n}-\theta^{\prime}\right)} \cdots \int_{0}^{2 \pi} \frac{d \theta_{j}}{2 \pi} \frac{\cos \left(\theta_{Q}-\theta_{j}\right)}{\cosh (\varphi)-\cos \left(\theta_{j}-\theta_{j+1}\right)} \cdots \\
& \times \int_{0}^{2 \pi} \frac{d \theta_{i}}{2 \pi} \frac{\cos \left(\theta_{Q}-\theta_{i}\right)}{\cosh (\varphi)-\cos \left(\theta_{i}-\theta_{i+1}\right)} \cdots \\
& \times \int_{0}^{2 \pi} \frac{d \theta_{1}}{\cosh (\varphi)-\cos \left(\theta_{1}-\theta_{2}\right)} \frac{1}{\cosh (\varphi)-\cos \theta_{1}} \\
& L_{i}(n)=\int_{0}^{2 \pi} \frac{d \theta_{n}}{2 \pi} \frac{1}{\cosh (\varphi)-\cos \left(\theta_{n}-\theta^{\prime}\right)} \cdots \int \frac{d \theta_{i}}{2 \pi} \frac{\cos ^{2}\left(\theta_{Q}-\theta_{i}\right)}{\cosh (\varphi)-\cos \left(\theta_{i}-\theta_{i+1}\right)} \cdots \\
& \times \frac{d \theta_{i}}{2 \pi} \frac{1}{\cosh (\varphi)-\cos \left(\theta_{1}-\theta_{2}\right)} \frac{1}{\cosh (\varphi)-\cos \theta_{1}} .
\end{aligned}
$$

Using Eqs. (A4) and (A2) we obtain for $I_{j}(n)$ in Eq. (A6)

$$
\begin{aligned}
I_{j}\left(n, \theta^{\prime}\right) & =\int_{0}^{2 \pi} \frac{d \theta_{j}}{2 \pi} \cos \left(\theta_{j}-\theta_{Q}\right) I_{0}\left(n-j, \theta_{j}-\theta^{\prime}\right) I_{0}\left(j-1, \theta_{j}\right) \\
& =\frac{\sinh [(n+1-j) \varphi] \sinh [j \varphi]}{\left[\sinh (\varphi)^{n+1}\right]} J_{2}\left[j \varphi,(n+1-j) \varphi, \theta^{\prime}, \theta_{Q}\right] \\
& =\frac{1}{[\sinh (\varphi)]^{n+1}} \frac{\sinh (n+1-j) \varphi \cos \theta_{Q}+\sinh (j \varphi) \cos \left(\theta_{Q}-\theta^{\prime}\right)}{\cosh [(n+1) \varphi]-\cos \theta^{\prime}},
\end{aligned}
$$

then

$$
\sum_{j=1}^{n} I_{j}\left(n, \theta^{\prime}\right)=\frac{1}{[\sinh (\varphi)]^{n+1}} \frac{1}{\cosh [(n+1) \varphi]-\cos \theta^{\prime}}\left[\cos \theta_{Q}+\cos \left(\theta_{Q}-\theta^{\prime}\right)\right] \frac{\sinh (n \varphi) \sinh [(n+1) / 2] \varphi}{\sinh (\varphi / 2)} .
$$

In the same way, we obtain from Eqs. (A8) and (A3) 


$$
\begin{aligned}
L_{i}(n)= & \int_{0}^{2 \pi} \frac{d \theta_{i}}{2 \pi} \cos ^{2}\left(\theta_{i}-\theta_{Q}\right) I_{0}\left(n-i, \theta_{i}-\theta^{\prime}\right) I_{0}\left(i-1, \theta_{i}\right) \\
= & \frac{\sinh [(n+1-i) \varphi] \sinh [i \varphi]}{[\sinh (\varphi)]^{n+1}} J_{3}\left[i \varphi ;(n+1-i) \varphi ; \theta^{\prime} ; \theta_{Q}\right] \\
= & \frac{1}{[\sinh (\varphi)]^{n+1}} \frac{1}{2} \frac{1}{\cosh [(n+1) \varphi]-\cos \theta^{\prime}} \\
& \quad \times\left\{\sinh (n+1) \varphi+e^{-i \varphi} \sinh [(n+1-i) \varphi] \cos 2 \theta_{Q}\right. \\
& \left.\quad+e^{-(n+1-i) \varphi} \sinh (i \varphi) \cos \left(2 \theta_{Q}-2 \theta^{\prime}\right)+2 e^{-(n+1) \varphi} \sinh (i \varphi) \sinh [(n+1-i) \varphi] \cos \left(2 \theta_{Q}-\theta^{\prime}\right)\right\} .
\end{aligned}
$$

The sums over $i$ are tedious but straightforward; then we get the result

$$
\begin{aligned}
\sum_{i=1}^{n} L_{i}(n)= & \frac{1}{[\sinh (\varphi)]^{n+1}} \frac{1}{\cosh [(n+1) \varphi]-\cos \theta^{\prime}} \frac{1}{2} \\
\times & \left\{\sinh [(n+1) \varphi]+\frac{1}{2}\left[\cos 2 \theta_{Q}+\cos \left(2 \theta_{Q}-2 \theta^{\prime}\right)\right]\left[\frac{\sinh (n \varphi)}{\sinh (\varphi)}-n e^{-(n+1) \varphi}\right]\right. \\
& \left.\quad+\cos \left(2 \theta_{Q}-\theta^{\prime}\right)\left[n e^{-(n+1) \varphi} \cosh [(n+1) \varphi]-e^{-(n+1) \varphi} \frac{\sinh (n \varphi)}{\sinh \varphi}\right] \cos \left(2 \theta_{Q}-\theta^{\prime}\right)\right\} .
\end{aligned}
$$

The calculations involving $I_{i j}(n)$ are the most difficult ones. We obtain from Eqs. (A7) and (A6)

$$
\begin{aligned}
I_{j i}(n) & =\int_{0}^{2 \pi} \frac{d \theta_{j}}{2 \pi} I_{0}\left(n-j, \theta_{j}-\theta^{\prime}\right) \cos \left(\theta_{j}-\theta_{Q}\right) I_{i}\left(j-1, \theta_{j}\right) \\
& =\frac{\sinh [(n+1-j) \varphi]}{[\sinh (\varphi)]^{(n+1-j)}} \int d \theta_{j} \frac{\cos \left(\theta_{j}-\theta_{Q}\right)}{\cosh [(n+1-j) \varphi]-\cos \left(\theta_{j}-\theta^{\prime}\right)} I_{i}\left(j-1, \theta_{j}\right)
\end{aligned}
$$

and, using Eq. (A9), together with Eqs. (A2) and (A3), we get

$$
I_{j i}(n)=\frac{1}{[\sinh (\varphi)]^{n+1}} \frac{1}{\cosh [(n+1) \varphi]-\cos \theta^{\prime}} \frac{1}{\sinh (j \varphi)} M_{j i},
$$

with

$$
\begin{aligned}
M_{j i}= & \sinh [(j-i) \varphi]\left\{\cos ^{2} \theta_{Q} \sinh [(n+1-j) \varphi]+\cos \theta_{Q}\left(\theta_{Q}-\theta^{\prime}\right) \sinh (j \varphi)\right\} \\
+\frac{\sinh (i \varphi)}{2}\{ & \sinh [(n+1) \varphi]+e^{-j \varphi} \sinh [(n+1-j) \varphi] \cos \left(2 \theta_{Q}\right) \\
& \left.+2 e^{-(n+1) \varphi} \sinh (j \varphi) \sinh [(n+1-j) \varphi] \cos \left(2 \theta_{Q}-\theta^{\prime}\right)+e^{-(n+1-j) \varphi} \sinh (j \varphi) \cos \left(2 \theta_{Q}-2 \theta^{\prime}\right)\right\} .
\end{aligned}
$$

Now we use the identity

$$
\cos (\alpha) \cos (\beta)=\frac{1}{2}[\cos (\alpha+\beta)+\cos (\alpha-\beta)]
$$

to recast Eq. (A14) in the form

$$
\begin{aligned}
M_{j i}=\sinh (j \varphi) \frac{1}{2}( & \sinh [(n+1-j+i) \varphi]+\cos \theta^{\prime} \sinh [(j-i) \varphi] \\
& +\cos \left(2 \theta_{Q}\right) e^{-i \varphi} \sinh [(n+1-j) \varphi]+\cos \left(2 \theta_{Q}-2 \theta^{\prime}\right) \sinh (i \varphi) e^{-(n+1-j) \varphi} \\
& \left.+\cos \left(2 \theta_{Q}-\theta^{\prime}\right)\left\{2 e^{-(n+1-j) \varphi} \sinh [(n+1-j) \varphi] \sinh (i \varphi)+\sinh [(j-i) \varphi]\right\}\right) .
\end{aligned}
$$

So the overall factor $\sinh (j \varphi)$ cancels the identical term in the denominator of Eq. (A13) and the sums over $i, j$ in Eq. (A5) can be easily performed.

First we observe that the two first terms in Eq. (A15) depend only on $n^{\prime}=j-i$; then we may write

$$
\begin{aligned}
\sum_{j=1}^{n} \sum_{n^{\prime}=1}^{j=1}\left\{\sinh \left[\left(n+1-n^{\prime}\right) \varphi\right]+\cos \theta^{\prime} \sinh \left(n^{\prime} \varphi\right)\right\} & =\sum_{n^{\prime}=1}^{n} \sum_{j=n^{\prime}+1}^{n}\left\{\sinh \left[\left(n+1-n^{\prime}\right) \varphi\right]+\cos \theta^{\prime} \sinh \left(n^{\prime} \varphi\right)\right\} \\
& =\sum_{n^{\prime}=1}^{n}\left(n-n^{\prime}\right)\left\{\sinh \left[\left(n+1-n^{\prime}\right) \varphi\right]+\cos \theta^{\prime} \sinh \left(n^{\prime} \varphi\right)\right\}
\end{aligned}
$$


and the sums in Eq. (A16) can be performed ${ }^{6}$ by using

$$
\sum_{n^{\prime \prime}} n^{\prime \prime} \sinh \left(n^{\prime \prime} \varphi\right)=\frac{\partial}{\partial \varphi} \sum_{n^{\prime \prime}} \cosh \left(n^{\prime \prime} \varphi\right) \text { and } \sum_{n^{\prime \prime}} n^{\prime \prime} \cosh \left(n^{\prime \prime} \varphi\right)=\frac{\partial}{\partial \varphi} \sum_{n^{\prime \prime}} \sinh \left(n^{\prime \prime} \varphi\right) \text {. }
$$

For the rest of the terms in Eq. (A16) one should sum first over $i$ up to $j-1$, and after, over $j$ from 1 to $n$. The procedure is standard but tedious, with the result

$$
\begin{aligned}
\sum_{j=1}^{n} \sum_{i=1}^{j=1} I_{j i}= & \frac{1}{[\sinh (\varphi)]^{n+1}} \frac{1}{\cosh [(n+1) \varphi]-\cos \theta} \frac{1}{\theta 2} \\
& \times\left[\frac{n}{2 \sin (\varphi / 2)}\left\{\cosh \left[\left(n+\frac{1}{2}\right) \varphi\right]-\cos \theta^{\prime} \cosh (\varphi / 2)\right\}\right. \\
& -\frac{\sinh (n \varphi / 2)}{2 \sinh ^{2}(\varphi / 2)}\left\{\cosh \left[\left[\frac{n}{2}+1\right] \varphi\right]-\cos \theta^{\prime}\left[\frac{n \varphi}{2}\right]\right\} \\
& +\frac{1}{2\left(e^{\varphi}-1\right)} \cos \left(2 \theta_{Q}\right)\left[\frac{2 \sinh [(n+1 / 2) \varphi] \sinh (n \varphi / 2)}{\sinh (\varphi / 2)}-\frac{\sinh (n \varphi)}{\sinh (\varphi)}+n e^{-n \varphi}\right] \\
& +\frac{1}{2\left(e^{\varphi}-1\right)} \cos \left(2 \theta_{Q}-\theta^{\prime}\right)\left[-n e^{-n \varphi} 2 \cosh [(n+1) \varphi]\right. \\
& \left.+\frac{1}{2\left(e^{\varphi}-1\right)} \cos \left(2 \theta_{Q}-2 \theta^{\prime}\right)\left[\frac{\sinh (n \varphi)}{\sinh (\varphi)}-\cot \left[\frac{\varphi}{2}\right]\left(1-e^{-n \varphi}\right)+n e^{-n \varphi}\right]\right]
\end{aligned}
$$

By introducing Eqs. (A4) (A10), (A11), and (A17) into Eq. (A5) we obtain Eq. (13).

\section{Calculation of Eq. (12) integrated over $\theta_{Q}$}

If we take advantage of the remark made in the text after Eq. (14) the algebra is simplified. We put

$$
\begin{aligned}
I^{\prime}{ }_{i, j}=\int \cdots \int & \frac{d \theta_{1}}{2 \pi} \frac{d \theta_{2}}{2 \pi} \cdots \frac{d \theta_{n}}{2 \pi} \\
& \times \frac{1}{\cosh \varphi-\cos \left(\theta_{1}-\theta\right)} \cdots \\
& \times \frac{1}{\cosh \varphi-\cos \left(\theta^{\prime}-\theta_{n}\right)} \cos \left(\theta_{i}-\theta_{j}\right) .
\end{aligned}
$$

Then

$$
\sum_{\substack{i, j=1 \\ i<j}} I_{i, j}^{\prime}
$$

can be written as a triangular table sum

$$
\begin{aligned}
\sum_{\substack{i, j=1 \\
i<j} I_{i, j}^{\prime}=I_{1,2}^{\prime}+I_{1,3}^{\prime}}^{\prime}+I_{1,4}^{\prime}+\cdots & +I_{1, n}^{\prime} \\
+I_{2,3}^{\prime}+I_{2,4}^{\prime}+\cdots & +I_{2, n}^{\prime} \\
+I_{3,4}^{\prime}+\cdots & +I_{3, n}^{\prime} \\
& \vdots \\
& +I_{n-1, n}^{\prime}
\end{aligned} .
$$

One easily verifies that all the terms within a given diagonal of the above triangle are equal, whatever the diagonal.

$$
\begin{aligned}
& I_{1,2}^{\prime}=I_{2,3}^{\prime}=\cdots=I_{n-1, n}^{\prime}, \\
& I_{1,3}^{\prime}=I_{2,4}^{\prime}=\cdots=I_{n-2, n}^{\prime}, \\
& I_{1, n-1}^{\prime}=I_{2, n}^{\prime} .
\end{aligned}
$$

Therefore,

$$
\sum_{\substack{i, j=1 \\ i<j}}^{n} I_{i, j}^{\prime}=\sum_{i=1}^{n}(n-i) I_{n-i, n}
$$

One can also note that within the last column, but only in this one, the terms cancel two by two, except the last one $I_{n-1, n}^{\prime}$, which, using (A20), allows some partial cancellations in other columns, too. However, this does not simplify things much compared to just computing (A21). $I_{n-i, n}^{\prime}$ can be written from (A18) with (A4) as follows: 


$$
\begin{array}{r}
I_{n-i, n}^{\prime}=\frac{1}{2 \pi} \int \frac{d \theta_{n}}{\cosh \varphi-\cos \left(\theta^{\prime}-\theta_{n}\right)} \frac{1}{2 \pi} \int \frac{d \theta_{n-1}}{\cosh \varphi-\cos \left(\theta_{n}-\theta_{n-1}\right)} \cdots \frac{1}{2 \pi} \int \frac{d \theta_{n-i} \cos \left(\theta_{n}-\theta_{n-i}\right)}{\cosh \varphi-\cos \left(\theta_{n-i+1}-\theta_{n-i}\right)} \\
\times I_{0}\left(l \equiv n-i-1, \theta^{\prime} \equiv \theta_{n-i}\right)
\end{array}
$$

Then, using (A1) and (A2) a number of times, one gets

$$
I_{n-i, n}^{\prime}=\frac{\sinh [(n-i+1) \varphi]+\sinh (i \varphi) \cos \theta^{\prime}}{\sinh ^{n+1} \varphi\left\{\cosh [(n+1) \varphi]-\cos \theta^{\prime}\right\}}
$$

Then, to perform (A21) we put $(n-i) \equiv n^{\prime \prime}$ so that

$$
\sum_{\substack{i, j=1 \\ i<j}}^{n} I_{i, j}^{\prime}=\frac{1}{\sinh ^{n+1} \varphi\left\{\cosh [(n+i) \varphi]-\cos \theta^{\prime}\right\}} \sum_{n^{\prime \prime}=1}^{n=1} n^{\prime \prime}\left\{\sinh \left[\left(n^{\prime \prime}+1\right) \varphi\right]+\sinh \left[\left(n-n^{\prime \prime}\right) \varphi\right] \cos \theta^{\prime}\right\}
$$

The sums in (A24) can be performed using

$$
\begin{aligned}
& \Sigma n^{\prime \prime} \sinh \left(n^{\prime \prime} \varphi\right)=\frac{\partial}{\partial \varphi} \Sigma \cosh \left(n^{\prime \prime} \varphi\right) \\
& \Sigma n^{\prime \prime} \cosh \left(n^{\prime \prime} \varphi\right)=\frac{\partial}{\partial \varphi} \Sigma \sinh \left(n^{\prime \prime} \varphi\right)
\end{aligned}
$$

The remaining sums are known ${ }^{6}$ and one gets

$$
\begin{aligned}
& \sum_{\substack{i, j=1 \\
i<j}}^{n} I_{i, j}^{\prime}=\frac{1}{\sinh ^{n+1} \varphi\left\{\cosh [(n+1) \varphi]-\cos \theta^{\prime}\right\}}\left\{\frac{n}{2 \sinh (\varphi / 2)}\left\{\cosh \left[\left(n+\frac{1}{2}\right] \varphi\right]-\cos \theta^{\prime} \cosh \frac{\varphi}{2}\right\}\right. \\
& \left.-\frac{\sinh (n \varphi / 2)}{2 \sinh ^{2}(\varphi / 2)}\left\{\cosh \left[\left(\frac{n}{2}+1\right] \varphi\right]-\cos \theta^{\prime} \cosh \left[\frac{n \varphi}{2}\right]\right\}\right] \text {. }
\end{aligned}
$$

Then, using (A4) and (A25) in (12) with the brackets $\{\cdots\}$ replaced by its average over $\theta_{Q}$,

$$
\left\{1-\frac{k_{F}^{2} Q^{2} \tau^{2}}{2} \sum_{\substack{i, j=1 \\ i<j}}^{n} \cos \left(\theta_{i}-\theta_{j}\right)-n \frac{k_{F}^{2} Q^{2} \tau^{2}}{2}\right\},
$$

we obtain

$$
\begin{aligned}
\left\langle\Gamma_{c}^{(n+1)}\left(\mathbf{k}, \mathbf{k}^{\prime} ; \widetilde{\omega}_{n+v}, \widetilde{\omega}_{n}\right)\right\rangle_{\theta_{Q}}= & \tau^{-1}\left[\frac{\tau^{-1}}{\omega_{v}+\tau^{-1}}\right]^{n} \sinh ^{(n+1)} \varphi\left\{\left[1-n \frac{k_{F}^{2} Q^{2} \tau^{2}}{2}\right] I\left(l \equiv n, \theta_{l+1} \equiv \theta^{\prime}\right)-\frac{k_{F}^{2} Q^{2} \tau^{2}}{2} \sum_{\substack{i, j=1 \\
i<j}}^{n} I_{i, j}^{\prime}\right\} \\
= & \tau^{-1}\left[\frac{\tau^{-1}}{\omega_{v}+\tau^{-1}}\right]^{n} \frac{1}{\cosh [(n+1) \varphi]} \\
& \times\left[\left[1-n \frac{k_{F}^{2} Q^{2} \tau^{2}}{2}\right] \sinh [(n+1) \varphi]\right. \\
& -\frac{k_{F}^{2} Q^{2} \tau^{2}}{2}\left[\frac{n}{2 \sinh (\varphi / 2)}\left[\cosh \left[\left(n+\frac{1}{2}\right) \varphi\right]-\cos \theta^{\prime} \cosh \left[\frac{\varphi}{2}\right]\right]\right. \\
& \left.-\frac{\sinh (n \varphi / 2)}{2 \sinh (\varphi / 2)}\left[\cosh \left[\left(\frac{n}{2}+1\right] \varphi\right]-\cos \theta^{\prime} \cosh \left[\frac{n \varphi}{2}\right]\right\}\right]
\end{aligned}
$$

which identifies with formula (13) integrated over $\theta_{Q}$. 


\section{APPENDIX B: CALCULATION OF SOME OF THE CONDUCTIVITY DIAGRAMS IN FIG. 3.}

In the following, we calculate the contribution of the diagrams in Fig. 3 to be $K_{(b)} \cdots$ for finite $\omega_{v}$; then, as usual, the conductivity formula will be obtained through

$$
\sigma=\lim _{\omega \rightarrow 0} \frac{K(i \omega)-K(0)}{i \omega}, \omega=i \omega_{v} .
$$

\section{Calculation of $K_{(b)}$}

$\mathbf{k}$ and $\mathbf{k}^{\prime}$ are the electron momenta on either side of the diffuson $\Gamma_{d}$ given in formula $(26) ; \widetilde{\omega}_{n+v}$ and $\left(\widetilde{\omega}_{n}\right)$ are the Matsubara frequencies of the upper (lower) electron lines. Then,

$$
\begin{aligned}
K_{(\mathrm{b})} & =-2 T \sum_{\omega_{n}} \iint \frac{d^{2} k}{(2 \pi)^{2}} \frac{d^{2} k^{\prime}}{(2 \pi)^{2}} \Gamma_{d} G\left(\mathbf{k}, \widetilde{\omega}_{n+v}\right) G\left(\mathbf{k}, \widetilde{\omega}_{n}\right) G\left(\mathbf{k}^{\prime}, \widetilde{\omega}_{n+v}\right) G\left(\mathbf{k}^{\prime}, \widetilde{\omega}_{n}\right) \frac{\mathbf{k} \cdot \mathbf{k}^{\prime}}{2} \\
& \approx-\frac{\omega_{v}}{\pi} \sum_{n=0}^{\infty} \tau^{-1}\left[\frac{\tau^{-1}}{\omega_{v}+\tau^{-1}}\right]^{n} \tau^{2} \int \frac{d \theta^{\prime}}{2 \pi} \frac{k_{F}^{2}}{2} \frac{\cos \theta^{\prime} \sinh [(n+1) \varphi]}{\cosh [(n+1) \varphi]}=-\frac{\omega_{v}}{\pi} \frac{k_{F}^{2}}{2} \tau e^{-\varphi} \sum_{n=0}^{\infty}\left[\frac{\tau^{-1} e^{-\varphi}}{\omega_{v}+\tau^{-1}}\right]^{n},
\end{aligned}
$$

which straightforwardly yields

$$
\sigma_{(\mathrm{b})}=\frac{k_{F}^{2} \tau}{2 \pi} \frac{1}{e^{\varphi}-1},
$$

which is formula (27) in the text.

\section{Calculation of $\boldsymbol{K}_{(\mathrm{c})}$}

Here the upper electron line carries momenta $\mathbf{k}$ and $\mathbf{k}^{\prime}$ on either side of the Cooperon $\Gamma_{c}$. The lower line carries $\mathbf{Q}-\mathbf{k}^{\prime}$ and $\mathbf{Q}-\mathbf{k}$. Momenta conservation imposes

$$
\mathbf{k}+\mathbf{k}^{\prime} \equiv \mathbf{Q}, \quad \mathbf{Q} \rightarrow 0
$$

Therefore $K_{(\mathrm{c})}$ is written as

$$
\begin{aligned}
K_{(\mathrm{c})} & =-2 T \sum_{\omega_{n}} \iint \frac{d^{2} \mathbf{k}}{(2 \pi)^{2}} \frac{d^{2} Q}{(2 \pi)^{2}} \Gamma_{c}(\mathbf{k},-\mathbf{k}) \frac{\mathbf{k} \cdot(\mathbf{Q}-\mathbf{k})}{2} G\left(\mathbf{k}, \widetilde{\omega}_{n+v}\right) G\left(Q-\mathbf{k}, \widetilde{\omega}_{n+v}\right) G\left(\mathbf{k}, \widetilde{\omega}_{n}\right) G\left(Q-\mathbf{k}, \widetilde{\omega}_{n}\right) \\
& =-2 T \sum_{\omega_{n}} \iint \frac{d^{2} \mathbf{k}}{(2 \pi)^{2}} \frac{d^{2} Q}{(2 \pi)^{2}} \Gamma_{c}(\mathbf{k},-\mathbf{k})\left[-\frac{k_{F}^{2}}{2}\right] G^{2}\left(\mathbf{k}, \widetilde{\omega}_{n+v}\right) G^{2}\left(\mathbf{k}, \widetilde{\omega}_{n}\right)
\end{aligned}
$$

where $\Gamma_{C}(\mathbf{k},-\mathbf{k})$ stands for

$$
\Gamma_{C}\left(\mathbf{k}, \mathbf{Q}-\mathbf{k}, \mathbf{Q} ; \widetilde{\omega}_{n+v}, \widetilde{\omega}_{n}\right),
$$

from which we deduce

$$
\sigma_{(\mathrm{c})}=-\frac{1}{\pi} \int \frac{Q d Q}{2 \pi}\left\langle\Gamma_{c}(k,-k)\right\rangle_{\theta_{Q^{\prime}, \omega_{v}=0}}\left(k_{F}^{2} \tau^{3}\right),
$$

which, with (23), yields

$$
\sigma_{(\mathrm{c})}=-\frac{1}{\pi^{2}} \frac{e^{\varphi}-1}{e^{\varphi}} \ln \left(\frac{L}{k_{F} \tau}\right), \quad \tau^{-1}=\frac{n_{I}}{\sinh \varphi},
$$

which is formula (28) is the text.

\section{Calculation of the other diagrams involving Cooperons}

The other diagrams can be computed the same way. We will thus get, compared with $\sigma_{(c)}$ in (B6),

$$
\sigma_{(\mathrm{e})}=-\frac{1}{\pi} \int \frac{Q d Q}{2 \pi}\left\langle\Gamma_{c}(\mathbf{k},-\mathbf{k})\right\rangle_{\theta_{\mathbf{Q}^{\prime}, \omega_{v}}=0}\left(k_{F}^{2} \tau^{3}\right) 2 \frac{1}{e^{\varphi}-1}=\sigma_{(\mathrm{c})} 2 \frac{1}{e^{\varphi}-1},
$$

which is formula (29) in the text; 


$$
\sigma_{(\mathrm{g})}=-\frac{1}{\pi} \int \frac{Q d Q}{2 \pi}\left\langle\Gamma_{c}(\mathbf{k},-\mathbf{k})\right\rangle_{\theta_{\mathbf{Q}, \omega_{v}}=0}\left(k_{F}^{2} \tau^{3}\right)\left(\frac{1}{e^{\varphi}-1}\right)^{2}=\sigma_{(\mathrm{c})}\left(\frac{1}{e^{\varphi}-1}\right)^{2},
$$

which identifies with (30), so that

$$
\sigma_{(\mathrm{c})}+\sigma_{(\mathrm{e})}+\sigma_{(\mathrm{g})}=\sigma_{(\mathrm{c})}\left[\frac{e^{\varphi}}{e^{\varphi}-1}\right]^{2} \text {. }
$$

On the other hand, we get

$$
\begin{aligned}
& K_{(\mathrm{d})}=-4 T \sum_{\omega_{n}} \iiint \frac{d^{2} \mathbf{k}}{(2 \pi)^{2}} \frac{d^{2} \mathbf{k}^{\prime}}{(2 \pi)^{2}} \frac{d^{2} \mathbf{Q}}{(2 \pi)^{2}} \Gamma_{c}\left(\mathbf{k}, \mathbf{k}^{\prime} ; \widetilde{\omega}_{n+v}, \widetilde{\omega}_{n}\right) G\left(\mathbf{Q}-\mathbf{k}^{\prime}, \widetilde{\omega}_{n+v}\right) G\left(\mathbf{k}, \widetilde{\omega}_{n+v}\right) G\left(\mathbf{k}^{\prime}, \widetilde{\omega}_{n+v}\right)\left(\mathbf{Q}-\mathbf{k}, \widetilde{\omega}_{n+v}\right) \\
& \quad \times G\left(\mathbf{Q}-\mathbf{k}^{\prime}, \widetilde{\omega}_{n}\right) G\left(\mathbf{Q}-\mathbf{k}, \widetilde{\omega}_{n}\right) n_{I} V^{2}\left(\mathbf{k}+\mathbf{k}^{\prime}-\mathbf{Q}\right) \frac{\left(\mathbf{Q}-\mathbf{k}^{\prime}\right) \cdot(\mathbf{Q}-\mathbf{k})}{2} \\
&=-\frac{2 \omega_{v}}{\pi} \sum_{n} \int \frac{Q d Q}{2 \pi} \int \frac{d \theta^{\prime}}{2 \pi}\left\langle\Gamma_{c}^{(n+1)}\left(k, k^{\prime}\right)\right\rangle_{\theta_{Q}, \omega_{v}=0}\left(-\frac{\tau^{3} k_{F}^{2}}{2}\right) \sinh \varphi \frac{\cos \theta^{\prime}}{\cosh \varphi+\cos \theta^{\prime}}
\end{aligned}
$$

and then

$$
\sigma_{(\mathrm{d})}=-\frac{1}{\pi} \sum_{n} \int \frac{Q d Q}{2 \pi} \int \frac{d \theta^{\prime}}{2 \pi}\left\langle\Gamma_{c}^{(n+1)}\left(k, k^{\prime}\right)\right\rangle_{\theta_{Q}, \omega_{v}=0}\left(k_{F}^{2} \tau^{3}\right) \sinh \varphi \frac{\cos \theta^{\prime}}{\cosh \varphi+\cos \theta^{\prime}},
$$

identical to (31) in the text.

Then one gets as well

$$
\begin{aligned}
& \sigma_{(\mathrm{f})}=\sigma_{(\mathrm{d})} 2 \frac{1}{e^{\varphi}-1}, \\
& \sigma_{(\mathrm{h})}=\sigma_{(\mathrm{d})} \frac{1}{\left(e^{\varphi}-1\right)^{2}},
\end{aligned}
$$

as indicated in the text in formulas (32) and (33). To calculate (B12), one has to use formula (13), where one performs first the integral over $\theta^{\prime}$. This is a lengthy but straightforward calculation, which yields

$$
\sigma_{(\mathrm{d})}=-\sigma_{(\mathrm{c})} e^{-\varphi} \text {. }
$$

Collecting the different contributions

$$
\sigma_{(\mathrm{d})}+\sigma_{(\mathrm{f})}+\sigma_{(\mathrm{h})}=-e^{-\varphi}\left(\frac{e^{\varphi}}{e^{\varphi}-1}\right)^{2} \sigma_{(\mathrm{c})},
$$

we finally obtain

$$
\begin{aligned}
\sigma_{\mathrm{loc}} & =\sigma_{(\mathrm{c})}+\sigma_{(\mathrm{d})}+\cdots+\sigma_{(\mathrm{h})} \\
& =\left(1-e^{-\varphi}\right)\left(\frac{e^{\varphi}}{e^{\varphi}-1}\right)^{2} \sigma_{(\mathrm{c})},
\end{aligned}
$$

which identifies with (37) using (28).
*Instituto de Fisica, Universidade Federal do Rio Grande do Sul, Caixa Postal 15051, 91500 Porto Alegre, Rio Grande do Sul, Brazil.

†Department of Physics, Clarkson University, Potsdam, N.Y. 13699.

${ }^{1}$ See, for instance, the review by P. A. Lee and T. V. Ramakrishnan, Rev. Mod. Phys. 57, 287 (1985).

${ }^{2}$ See, for instance, L. D. Landau and E. M. Lifshitz, Quantum Mechanics (Pergamon, New York, 1959).

${ }^{3}$ M. E. Fisher, S. K. Ma, and B. G. Nickel, Phys. Rev. Lett. 29, 917 (1972); J. Sak, Phys. Rev. B 8, 281 (1973).

${ }^{4}$ See, for instance, A. A. Abrikosov, L. P. Gorkov, and I. E.
Dzialoshinski, Methods of Quantum Field Theory in Statistical Physics (Prentice-Hall, Englewood Cliffs, NJ, 1963).

${ }^{5}$ S. V. Maleev and B. T. Toperverg, Zh. Eksp. Teor. Fiz. 69, 1440 (1975) [Sov. Phys. JETP 42, 734 (1976)].

${ }^{6}$ See, for instance, I. S. Gradshteyn and I. W. Ryzhik, Tables of Integrals, Series and Products (Academic, New York, 1965).

${ }^{7}$ A. Weinrib and B. I. Halperin, Phys. Rev. B 27, 413 (1983).

${ }^{8}$ M. C. Varriale and A. Theumann, J. Phys. A 23, L719 (1990).

${ }^{9}$ S. Hikami and E. Brézin, J. Phys. (Paris) 46, 2021 (1985).

${ }^{10}$ S. John and M. J. Stephen, Phys. Rev. B 28, 6358 (1983).

${ }^{11}$ M. T. Béal-Monod and G. Forgacs, Phys. Rev. B 45, 3971 (1992). 